\title{
The CITIDEMAGE Study: Combined Treatment with a Cholinergic Precursor in Dementia Patients
}

Pietro Gareri ${ }^{1,}{ }^{*}$, Antonino Maria Cotroneo ${ }^{2}$, Giuseppe Orsitto ${ }^{3}$, Nicola Veronese ${ }^{4}$, Salvatore Putignano $^{5}$

1. Center for Cognitive Disorders and Dementia-Catanzaro Lido, ASP Catanzaro, Catanzaro, Italy; EMail: pietro.gareri@alice.it

2. Department of Elderly Health Care-Birago di Vische Hospital and Botticelli Territorial GeriatricsASL TO 2, Turin, Italy; E-Mail: geriatrix1@libero.it

3. U.O.C. Internal Medicine Ward, P.O. Bari Sud "Di Venere", Bari-Azienda Sanitaria Locale di Bari, Italy; E-Mail: giuseppeorsitto@katamail.com

4. Geriatric Unit, Department of Internal Medicine and Geriatrics, University of Palermo, Palermo, Italy; E-Mail: ilmannato@gmail.com

5. Operative Unit Elderly Care, District 30, ASL Napoli Centro, Italy; E-Mail: dott.s.putignano@alice.it

* Correspondence: Pietro Gareri; E-Mail: pietro.gareri@alice.it

Academic Editor: R. M. Damian Holsinger

Special Issue: $\underline{\text { Aging and Alzheimer's Disease }}$

OBM Geriatrics

2022, volume 6 , issue 1

doi:10.21926/obm.geriatr.2201193
Received: September 12, 2021

Accepted: February 27, 2022

Published: March 08, 2022

\begin{abstract}
Certain previous studies have demonstrated the benefits of the addition of citicoline, a cholinergic precursor, to acetylcholinesterase inhibitors (AchEls) or memantine in patients with Alzheimer's disease (AD). The present study showed the effectiveness of oral citicoline plus AchEls plus memantine in outpatients with $A D$. This was a retrospective case-control study involving 169 patients aged 65 years old or older having AD (mean age: $78.7 \pm 4.9$ years). In addition, 84 patients were treated with AchEls plus memantine plus citicoline $1 \mathrm{~g} /$ day given orally (group A), and 85 patients were treated with AchEls plus memantine (group B). In both
\end{abstract}

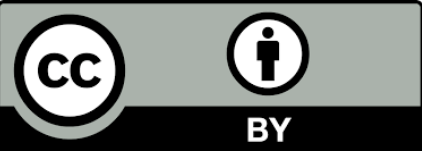

(C) 2022 by the author. This is an open access article distributed under the conditions of the Creative Commons by Attribution License, which permits unrestricted use, distribution, and reproduction in any medium or format, provided the original work is correctly cited. 
groups, memantine and AchEls were used at the highest dosage tolerated. Tests were administered at baseline (TO), after 6 (T1), and 12 months (T2) and included MMSE, ADL, IADL, $\mathrm{NPI}, \mathrm{CIRS}$, GDS-short form, the EuroQoL, and the Sleep questionnaire. The primary outcomes were the effects of combined treatment versus AchEls plus memantine on cognitive functions assessed by MMSE. The secondary outcomes were possible side effects of treatment in both groups, the influence on daily life functions, behavioral symptoms, quality of life, and sleep. Patients in group A showed a mild increase in MMSE at 6 (15.85 \pm 2.86 vs. $16.39 \pm 2.93)$ and 12 months ( $16.39 \pm 2.93$ vs. $16.43 \pm 3.08)$. On comparing the two groups, the difference in MMSE score was significant, both at T1 $(p=0.003)$ and T2 $(p=0.011)$. Moreover, a significant improvement in GDS and EuroQoL scores was observed. No differences in secondary outcomes, including side effects, were observed between the two groups. This study strengthens the role of citicoline plus AchEls plus memantine in patients with AD.

\section{Keywords}

Older people; Alzheimer's disease; cholinergic hypothesis; citicoline; memantine; acetylcholinesterase inhibitors; quality of life; cognition

\section{Introduction}

In the majority of countries, the population is rapidly becoming older; as a consequence, the prevalence of age-related disorders has been increasing. Alzheimer's disease (AD) is the most common form of dementia; its prevalence rate increases exponentially with age, particularly increasing markedly after 65 years and accounting for 60 to $80 \%$ of cases. Between the age of 60 and 85 years, a 15-fold increase in the prevalence of dementia, mainly AD, was assessed [1].

The prevalence of AD appears to be considerably higher in the US when compared with Africa, Asia, and Europe, even if it may relate to the methods of diagnosis. About 5.8 million Americans aged 65 years and older were found to be affected with AD in 2020, and $80 \%$ were 75 years old or older [2]. According to future projections, the number of people with a diagnosis of AD will increase up to 13.8 million individuals in 2050 [3].

None of the pharmacologic medications available today for $A D$ can slow or prevent the destruction of neurons that cause Alzheimer's symptoms. Drugs approved for the treatment of AD include acetylcholinesterase inhibitors (AchEls) rivastigmine, galantamine, donepezil, and the $\mathrm{N}$ methyl-D-aspartate (NMDA) receptor antagonist memantine. The Food and Drug Administration (FDA) also approved a combination of memantine and donepezil. AchEls are widely used in patients with $A D$ and have shown symptomatic efficacy [4-6]. They temporarily improve the cognitive symptoms by increasing the amount of acetylcholine in the brain. Memantine blocks NMDA receptors in the brain from excess stimulation that can damage the nerve cells. The effectiveness of these drugs varies from individual to individual and is limited in duration.

Therefore, AChEls and memantine may have a complementary and synergistic action in the treatment of $A D$ [7]. As described above, memantine and AChEls target two different pathological pathways, i.e., the dysfunctional glutamatergic and cholinergic transmitter systems, respectively. In addition, preclinical studies have demonstrated that memantine has neuroprotective effects 
because it can prevent glutamatergic overstimulation and the consequent neurotoxicity, and prevent the cholinergic fiber loss in the parietal cortex $[8,9]$. In particular, it inhibits calcium influx into cells that are normally caused by chronic NMDA receptor activation by glutamate [9].

Neuroinflammation is the first step in neurodegeneration as well as in cognitive impairment; it is characterized by non-neuronal cells activation (microglia and astrocytes), leading to toxic mediator release (i.e., reactive oxygen species [ROS], inflammatory cytokines) [10]. However, the final target is represented by the cholinergic neuron; indeed, the cholinergic system is one of the primary system's failures that contribute to the pathogenesis of $A D$ [11]. Furthermore, the role of cholinergic precursors in combination therapy with cholinesterase inhibitors has opened a new possible therapeutic option for the treatment of $A D$. In contrast, up to now, we do not have any drugs against this disease other than cholinesterase inhibitors and memantine. Aducanumab was recently approved by the Food and Drug Administration (FDA), even if its true efficacy is somewhat controversial [12].

The ASCOMALVA study was a trailblazer, showing the efficacy of donepezil plus choline alphoscerate [13]. However, we have shown the effects of another cholinergic precursor, citicoline. Some years ago, we had reported the benefits on cognition of citicoline $1 \mathrm{~g}$ plus AchEls (The CITIRIVAD and the CITICHOLINAGE studies) $[14,15]$ and recently of citicoline $1 \mathrm{~g}$ plus memantine (CITIMEM study) [16]. Recent retrospective studies have demonstrated the effectiveness of citicoline in a combined treatment with AchEls and memantine $[17,18]$. Citicoline exerts beneficial effects both in degenerative and vascular cognitive decline; it works through an increase in acetylcholine intra-synaptic levels and by promoting phospholipid synthesis (chiefly phosphatidylcholine), cellular function, and neuronal repair $[15,16]$.

Therefore, on the route traced by our previous studies, we have postulated that coadministration of AchEls, memantine, and citicoline exert a synergistic action on patients with AD.

The present study showed the effectiveness of oral citicoline plus AchEls plus memantine in outpatients with AD. This study is called the CITIDEMAGE study (CITIcoline in combination treatment in DEMentia AGEd patients).

\section{Patients and Methods}

\subsection{Sample Selection}

This was a retrospective case-control multicentric study performed between 2016 and 2018 on 169 outpatients with AD aged 65 years old or older (mean age: $78.3 \pm 5.68$ years old). The study involved four different centers for dementia all over Italy.

Patients affected with $A D$ and treated with combination therapy (citicoline + memantine + an AchEl) or memantine + AchEl were randomly selected in a 3-year time range from an original sample of 320 patients.

The inclusion criteria are reported below:

-Age of 65 years or older;

-People affected with AD;

-People had to be on treatment with memantine + AchEl or the combination therapy with citicoline sodium salt $1000 \mathrm{mg} /$ day for at least 3 months, from the scheduled start.

The exclusion criteria were: 
-Patients with other neurological disorders or taking medications potentially able to interfere with the results of the study (for example, drugs used for cognitive impairment, i.e., cognitive enhancers such as choline alphoscerate, choline bitartrate, L- $\alpha$-glycerophosphorylethanolamine, homotaurine).

Eighty-four patients (39 women, 45 men), mean age $77.7 \pm 5.47$ years old, with an AchEl plus memantine plus citicoline $1 \mathrm{~g} /$ day given orally (group A, case), 85 patients (47 women, 38 men), mean age $78.8 \pm 5.89$, were treated with an AchEl plus memantine (group $B$, controls).

In both groups, memantine and AchEls were used the highest dosage tolerated. Memantine tablets 10 and $20 \mathrm{mg}$ were used; among the AchEls, we included only patients with donepezil (oral tablets 5 and $10 \mathrm{mg}$ ) and rivastigmine (transdermal patches: 4.6, 9.5, and $13.3 \mathrm{mg}$ ) because of the reduced availability of galantamine.

Diagnosis of $A D$ has been made according to clinical symptoms, neuropsychological tests assessing different cognitive areas, such as memory, language, praxis (i.e., Mini-Mental State Examination, Montreal Canadian Assessment, IWG-2 (International Working Group-2) criteria [19], the presence of atrophy of the medial temporal lobe on brain imaging (CT scan or MRI scan) and/or, if needed, hypometabolism of the temporoparietal cortex, and posterior cingulate/precuneus cortex on 18-fluorodeoxyglucose-PET (FDG PET) [19].

The research was in accordance with national requirements and conform to the principles embodied in the 1964 Declaration of Helsinki (http:/www.wma.net) as well as to the International Ethical Guidelines for Biomedical Research Involving Human Subjects and the International Guidelines for Ethical Review for Epidemiological Studies (http:/www.cioms.ch). Written informed consent was obtained from all patients, or their legal representatives.

\subsection{Tests}

Cognitive functions were assessed by MMSE [20], daily life functions by Activities of Daily Living (ADL) and Instrumental ADL (IADL) [21, 22], behavioral symptoms by Neuropsychiatric Inventory Scale (NPI) [23], comorbidities by CIRS (comorbidity index) [24], and mood by Geriatric Depression Scale (GDS)-short form [25]. In addition, the Euro quality of life (EuroQoL-5D) [26] and the Sleep questionnaire were used [27]. EuroQoL-5D describes the health-related quality of life by reporting five dimensions, including mobility, self-care, usual activities, pain/discomfort, and anxiety/depression. It also includes a Visual Analog Scale (VAS), reporting the perceived health status ranging from 0 to 100 (respectively the worst and the best possible health status) [26]. The Sleep questionnaire is an 8-item screening tool reporting concerns about getting to sleep, remaining asleep, sleep quality, daytime personal functioning, daytime performance, duration of sleep problem, nights per week having a sleep problem, and extent troubled by poor sleep [27].

Tests were administered at baseline (T0), after 6 (T1), and 12 months (T2).

\subsection{Outcomes}

The primary outcomes were:

-Effects of combined treatment versus AchEls plus memantine alone on cognitive functions assessed by MMSE.

The secondary outcomes were:

-Possible side effects of combination therapy versus AchEls plus memantine alone; 
-Influence on daily life functions, mood, and behavioral symptoms, sleep, and quality of life.

Informed consent was signed by all participants or their legal representatives for using their data and medical records.

The study protocol was approved by the Ethics Committee (Italy, Calabria Region, registered protocol on June 21, 2018).

\subsection{Statistical Analysis}

Data are expressed as mean \pm standard deviation (SD). Student's $t$-test or Chi-square test, as appropriate, was used for performing comparisons among groups. Repeated measure analysis of variance (ANOVA) was applied to assess the differences in changes between data values at baseline. ANOVA was also used for assessing the difference between the two groups at 6 (T1) and 12 (T2) months. Significant differences were assumed to be present at $p<0.05$.

Statistical Package for the Social Sciences (SPSS) software, program version 23.0 for Windows, was used for performing all analyses.

\section{Results}

Age, gender, education, as well as different tests used, MMSE, CIRS, ADL, IADL, NPI, GDS-short form, and EuroQoL at baseline were not significantly different at baseline (Table 1).

Table 1 Main characteristics at baseline in the population studied (mean age, gender, education, test scores, comorbidities, and drugs used).

\begin{tabular}{|c|c|c|c|c|}
\hline & $\begin{array}{l}\text { CASE } \\
\text { Combined Treatment } \\
\text { (Group A) }\end{array}$ & $\begin{array}{l}\text { CONTROL } \\
\text { Memantine+AchEls } \\
\text { (Group B) }\end{array}$ & $\mathbf{P}$ & ALL POPULATION \\
\hline $\mathbf{n}$ & 84 & 85 & & 169 \\
\hline Age, years & $77.7 \pm 5.47$ & $78.8 \pm 5.89$ & 0.21 & $78.3 \pm 5.68$ \\
\hline \multicolumn{5}{|l|}{ Gender } \\
\hline - Men & 45 (53.5\%) & $38(44.7 \%)$ & & $83(49.1 \%)$ \\
\hline - Women & 39 (46.5\%) & $47(55.3 \%)$ & & 86 (50.9\%) \\
\hline Education, years & $6.84 \pm 4.02$ & $6.87 \pm 4.1$ & 0.96 & $6.85 \pm 4.06$ \\
\hline CIRS & $3.61 \pm 1.08$ & $3.34 \pm 1.2$ & 0.12 & $3.35 \pm 1.17$ \\
\hline MMSE & $15.85 \pm 2.86$ & $15.11 \pm 3$ & 0.10 & $15.47 \pm 2.97$ \\
\hline ADL & $3.45 \pm 1.1$ & $3.38 \pm 1.05$ & 0.67 & $3.41 \pm 1.08$ \\
\hline
\end{tabular}




\begin{tabular}{lllll} 
IADL & $2.29 \pm 1.2$ & $2.36 \pm 1.07$ & 0.68 & $2.33 \pm 1.13$ \\
NPI & $13.46 \pm 8.54$ & $13.5 \pm 8.46$ & 0.97 & $13.48 \pm 8.5$ \\
GDS (5 items) & $3.35 \pm 0.94$ & $3.34 \pm 0.91$ & 0.94 & $3.34 \pm 0.92$ \\
EuroQoL & & & & \\
& & & & \\
Sleep Questionnaire & $19.52 \pm 1.73$ & $53.65 \pm 9.69$ & 0.99 & $53.35 \pm 9.82$ \\
Comorbidities & & & & \\
Hypertension & $63(75 \%)$ & $19.18 \pm 1.76$ & 0.203 & $19.35 \pm 1.75$ \\
Osteoarthritis & $64(80.9 \%)$ & $65(76.4 \%)$ & & \\
Heart disease & $31(36.9 \%)$ & $70(82.3 \%)$ & 0.93 & $128(75.7 \%)$ \\
$\begin{array}{l}\text { Diabetes } \\
\text { COPD }\end{array}$ & $26(30.9 \%)$ & $34(40 \%)$ & 0.78 & $65(38.4 \%)$ \\
BPSD & $13(15.4 \%)$ & $15(17.6 \%)$ & 0.87 & $51(30.1 \%)$ \\
Drugs & $12(14.2 \%)$ & $13(15.2 \%)$ & 0.74 & $28(16.5 \%)$ \\
Cardiovascular drugs & $71(84.5 \%)$ & & 0.87 & $25(14.7 \%)$ \\
NSAIDs & $38(45 \%)$ & $70(82.5 \%)$ & & \\
Antidiabetics & $26(30.9 \%)$ & $34(48 \%)$ & 0.90 & $141(83.4 \%)$ \\
Antidepressants & $9(10.7 \%)$ & $24(28.2 \%)$ & 0.66 & $72(42.6 \%)$ \\
Antipsychotics & $7(8.3 \%)$ & $10(11.7 \%)$ & 0.77 & $50(29.5 \%)$ \\
Others & $8(9.5 \%)$ & $8(9.4 \%)$ & 0.84 & $19(11.2 \%)$ \\
\hline
\end{tabular}

$A D=$ Alzheimer's Disease; $A D L=$ Activities of Daily Living; $B P S D=$ Behavioral and Psychiatric Symptoms of Dementia; Cardiovascular drugs = antihypertensive drugs, antiaggregants, diuretics, nitrates, $\beta$-blockers, digoxin; CIRS $=$ Cumulative IIIness Rating Scale; COPD = Chronic Obstructive Pulmonary Disease; EuroQoL = Euro quality of life; GDS = Geriatric Depression Scale; $\mathrm{IADL}=$ Instrumental Activities of Daily Living; $\mathrm{MMSE}$ = Mini Mental State Examination; NPI = Neuropsychiatric Inventory; NSAIDs = non-steroidal anti-inflammatory drugs.

Table 1 reports the main characteristics at baseline in the population included. Interestingly, no differences were recorded at baseline between Group A and Group B.

Overall, 22 patients (13.1\%) were treated with memantine $10 \mathrm{mg}$ and 147 of them (86.9\%) with memantine $20 \mathrm{mg} ; 22$ patients (13.1\%) were treated with donepezil $5 \mathrm{mg}$ and 51 of them (30.1\%) were taking donepezil $10 \mathrm{mg}$. Rivastigmine patch $9.5 \mathrm{mg}$ was used in 66 patients (39.1\%) and 13.3 mg was used in 30 patients (17.7\%).

Patients treated with citicoline plus AchEls plus memantine showed a mild but non-significant increase in MMSE between T0 and T1 (15.85 \pm 2.86 vs. $16.39 \pm 2.93$ ) and between T1 and T2 (16.39 \pm 2.93 vs. $16.43 \pm 3.08$ ). No differences in gender were found.

The difference in MMSE score was significant when Group A was compared with Group B over time, both at T1 $(p=0.003)$ and T2 $(p=0.011)$ (Figure 1$)$. 
Figure 1

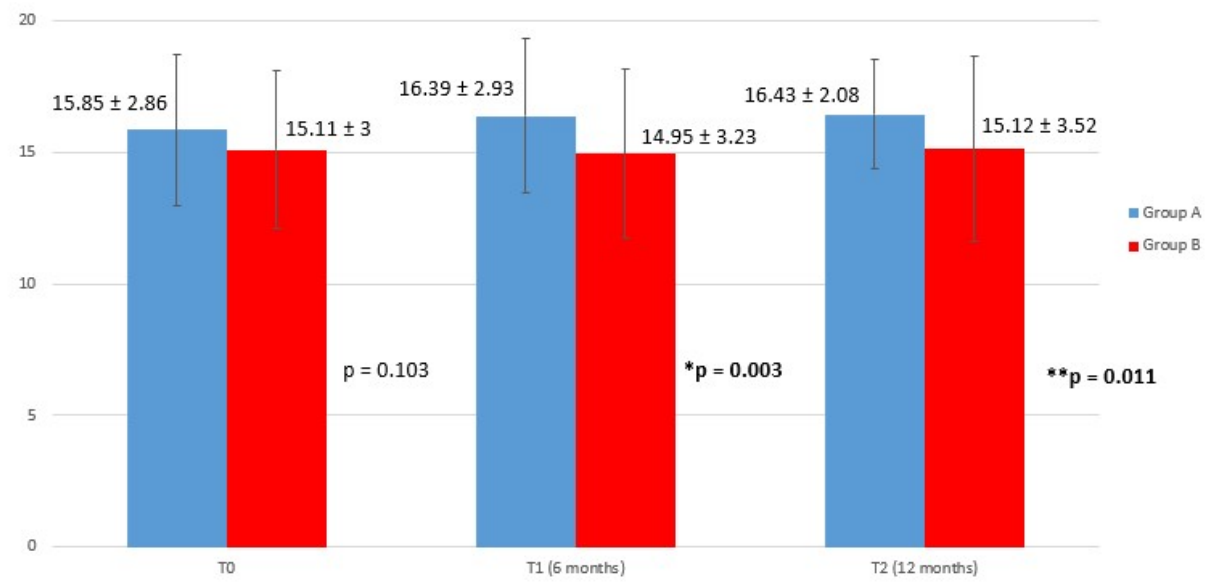

MMSE

Figure 1 Inter-group changes in MMSE score over time are shown.

There was also a significant improvement in GDS in patients taking the combined treatment with citicoline ( $p=0.000$ at T1, 0.008 at T2), but not between T1 and T2 ( $p=0.37$ ) (Figure 2) and in EuroQoL scores both at T1 $(p=0.05)$ and T2 $(\underline{p}=0.000)$ (Figure 3$)$ in patients taking the combined treatment.

Figure 2

GDS

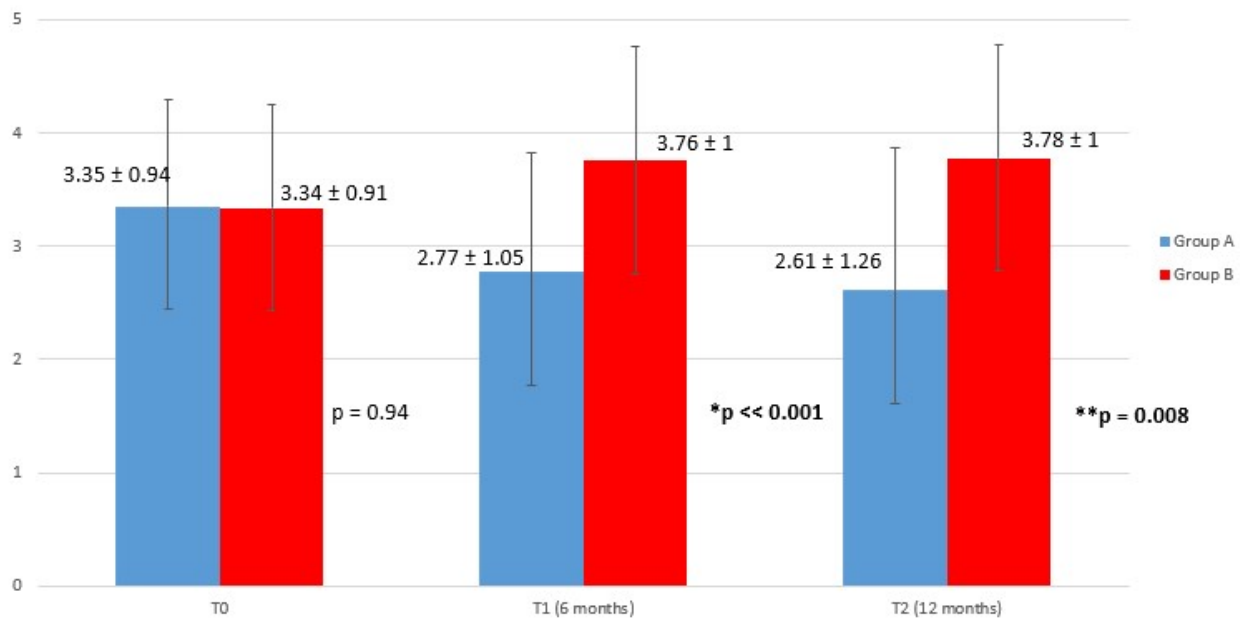

Figure 2 GDS scores. A significant improvement in GDS score was found at T1 and T2. 
Figure 3



EuroQol

Figure 3 Improvement in the EuroQoL score at 6 and 12 months in patients taking the combined treatment.

No significant intra-and inter-group changes were found on $A D L, I A D L, N P I$, and sleep questionnaire over time, as shown in Table 2, or GDS and EuroQoL (Table 3).

Table 2 Changes in test scores (MMSE, GDS-5 items, EuroQoL) over time (T0, T1, and T2).

\begin{tabular}{llll}
\hline Tests & T0 & T1 & T2 \\
\hline CIRS & & & \\
Case & $3.61 \pm 1.08$ & $3.63 \pm 1.08$ & $3.63 \pm 1.08$ \\
Controls & $3.34 \pm 1.2$ & $3.08 \pm 1.2$ & $3.08 \pm 1.2$ \\
MMSE & & & \\
Case & $15.85 \pm 2.86$ & $16.39 \pm 2.93$ & $16.43 \pm 3.08$ \\
Controls & $15.11 \pm 3$ & $14.95 \pm 3.23^{*}$ & $15.12 \pm 3.52^{*}$ \\
ADL & & & \\
Case & $3.45 \pm 1.1$ & $3.30 \pm 0.99$ & $3.10 \pm 1.06$ \\
Controls & $3.38 \pm 1.05$ & $3.33 \pm 1.08$ & $3.17 \pm 1.03$ \\
IADL & & & \\
Case & $2.29 \pm 1.2$ & $2.33 \pm 0.94$ & $2.38 \pm 1.19$ \\
Controls & $2.36 \pm 1.07$ & $2.35 \pm 1.02$ & $2.22 \pm 0.96$ \\
NPI & & & \\
Case & $13.46 \pm 8.54$ & $11.75 \pm 9.14$ & $10.82 \pm 10.27$ \\
Controls & $13.5 \pm 8.46$ & $13.57 \pm 8.17$ & $13.46 \pm 7.39$ \\
GDS (5 items) & & & \\
Case & $3.36 \pm 0.94$ & $2.77 \pm 1.05^{*}$ & $2.61 \pm 1.26^{*}$
\end{tabular}




$\begin{array}{lccc}\text { Controls } & 3.34 \pm 0.91 & 3.76 \pm 1^{* *} & 3.78 \pm 1^{* *} \\ \begin{array}{l}\text { EuroQoL } \\ \text { Case }\end{array} & 53.66 \pm 9.92 & 56.71 \pm 10.08^{*} & 58 \pm 11.39^{*} \\ \text { Controls } & 53.65 \pm 9.69 & 48.3 \pm 9.04^{* *} & 48.3 \pm 8.95^{* *} \\ \text { Sleep Questionnaire } & & & \\ \text { Case } & 19.52 \pm 1.73 & 19.66 \pm 1.32 & 19.66 \pm 1.32 \\ \text { Controls } & 19.18 \pm 1.76 & 19.33 \pm 1.37 & 19.33 \pm 1.97\end{array}$

CIRS = Cumulative Illness Rating Scale; MMSE = Mini Mental State Examination; ADL = Activities of Daily Living; IADL = Instrumental Activities of Daily Living; NPI = Neuropsychiatric Inventory; GDS-5 items = Geriatric Depression Scale 5 items; EuroQoL = Euro quality of Life. ${ }^{*} p<0.05$. Note that controls worsen in MMSE, GDS and EuroQoL tests at T1 and T2.

Table 3 No differences in side effect rate were recorded between Group A and Group B.

\begin{tabular}{lll}
\hline Kind of side effects & Case $\mathbf{n}(\%)$ & Control $\mathbf{n}(\%)$ \\
\hline Irritability & $4(4.76 \%)$ & $1(1.17 \%)^{*}$ \\
Restlessness & $1(1.19 \%)$ & $2(2.35 \%)$ \\
Epigastralgia & $3(3.57 \%)$ & $3(3.52 \%)$ \\
Diarrhea & $1(1.19 \%)$ & $2(2.35 \%)$ \\
Constipation & $1(1.19 \%)$ & $1(1.17 \%)$ \\
Dizziness & $1(1.19 \%)$ & $2(2.35 \%)$ \\
Itch & $1(1.19 \%)$ & $1(1.17 \%)$ \\
Overall $(24)$ & $12(14.2 \%)$ & $12(14.1 \%)$ \\
\hline
\end{tabular}

Significant differences were found in MMSE, GDS-5 items, EuroQoL between Group A and Group B. Interestingly, regarding MMSE, a non-significant increase was found at T1 and T2 in Group A. A significant difference was found when comparing Group A and Group B.

Regarding GDS and EuroQoL, a significant decrease was recorded in Group A (patients taking the triple therapy) at T1 and T2 (this was indicated by the single asterisk *). The difference was highly evident when comparing Group A and Group B (that is patients taking the triple therapy vs. those taking memantine plus an AchEl) at T1 and T2 (this was indicated by the double-asterisk ${ }^{* *}$ ).

Side effects were few and superimposable in the two groups, as reported in Table 3 . No differences in the side effects rate were recorded between Group A and Group B (14.2\% vs. 14.1\%, respectively). The main side effects reported were irritability, restlessness, epigastralgia, diarrhea, constipation, dizziness, and itch (Table 3).

\section{Discussion}

As previously stated, the potential role of citicoline in a combination treatment with AchEls and memantine is worth investigating [16]. We conducted this study and preliminary results were presented a few months ago at the 2020 AAIC (Alzheimer's Association International Conference) [28]. 
The CITIDEMAGE study showed that a combined treatment of citicoline $1 \mathrm{~g}$ plus AchEls plus memantine led to a mild but non-significant increase in MMSE at T1 and T2. Interestingly, the difference in the MMSE score was significant when cases and controls were compared over time, both at $\mathrm{T} 1$ and $\mathrm{T} 2$.

Furthermore, the benefits of combined treatment of AchEls plus memantine with citicoline vs. AchEls plus memantine alone were found on GDS and EuroQoL scores. Indeed, there was a significant improvement in the mood and quality of life perceived and a significant difference in the same scores in the control group at 6 and 12 months. The effects of the triple therapy on cognition, mood, and EuroQoL could be due to the synergistic action of citicoline, AchEls, and memantine; if on the one hand AchEls plus memantine exert an effective action on cognition through different mechanisms, the add-on therapy with citicoline, on the other hand, has a remarkable effect on cholinergic, noradrenergic, and dopaminergic neurotransmission, and the synthesis of serotonin via S-adenosyl-methionine (see the following text for details). We believe that the first-ever reported effects on the quality of life could be the consequence of an improved mood.

Some years ago, the Memage Study showed that memantine plus AchEls slowed down cognitive impairment and decreased agitation and aggression [9]. This is in line with other studies showing the effects of these drugs acting on glutamatergic and cholinergic pathways, respectively [4-8]. Glutamate plays a key role in cognition and the neurobiology of anxiety [29, 30]. The add-on treatment with a cholinergic precursor, such as citicoline, is advantageous, especially over a long time (6-12 months) because it can increase acetylcholine levels and protect neuronal membranes. Moreover, it has several remarkable actions reported in Table 4 [16, 31-45].

Table 4 Role of citicoline, an old drug with pleiotropic mechanisms [16, 31-45].

\section{CITICOLINE:}

-at the biochemical and cell level

- boosts acetylcholine synthesis [31-34]

- enhances phosphatidylcholine, phosphatidylserine, and phosphatidylethanolamine synthesis [31-34]

- inhibits apoptosis associated with cerebral ischemia by preventing caspase-3 activation [31-33]

- prevents the generation of free radicals at the site of ischemia and accumulation of free fatty acids [31-33]

- prevents the loss of cardiolipin, thus improving mitochondria energy metabolism [31-34]

- increases noradrenaline and dopamine levels in the CNS [33-35]

- promotes neuroprotection during hypoxia and ischemia [33-38]

- improves learning and memory performance in animal models of brain aging and promotes restoration of the membrane $\mathrm{Na}^{+} / \mathrm{K}^{+}$ATPase and the mitochondrial ATPase [31-33]

- inhibits phospholipase $A_{2}$ activation; thus, accelerating the reabsorption of cerebral edema in experimental models [32, 33]

- increases SIRT1 protein expression in the rat brain, cultured neurons, and circulating blood mononuclear cells $[36,39,40]$

- increases the activity of blood serum AChE, BChE, and NEP [40]

- decreases and delays neuronal glutamate efflux, stimulates glutathione synthesis, promotes neurogenesis, gliogenesis, and synaptogenesis [41-43] 
-at the clinical level

- is effective in cognitive impairment of diverse etiology, $A D, M D, P D, V D, V C l$, glaucoma, amblyopia, head trauma [33-35, 44, 45]

- improves the immediate and delayed recall of words and objects, verbal, short, and long-term memory [44]

- slows down cognitive impairment in people with $A D$ or $M D$ treated with AchEls (the CITICHOLINAGE Study) [15]

- slows down cognitive impairment in people affected with $A D$ or MD treated with memantine (the CITIMEM study) [16]

$\mathrm{AChE}=$ acetylcholinesterase $; \mathrm{BChE}=$ butyrylcholinesterase; $\mathrm{AD}=$ Alzheimer's disease; $\mathrm{MD}=$ mixed dementia; NEP = neprilysin; $\mathrm{PD}=$ Parkinson's disease; $\mathrm{VCl}$ = vascular cognitive impairment; $\mathrm{VD}=$ vascular dementia.

In contrast, we have already demonstrated the role of combined treatment of citicoline $1 \mathrm{~g}$ with memantine [16] or AchEls [14, 15], respectively. In particular, the CITIMEM Study was the first study assessing the efficacy of citicoline and the $\mathrm{N}$-methyl-D-aspartate (NMDA) receptor antagonist memantine in significantly slowing down the progression of disease in $A D$ and MD patients vs. memantine alone [16]. The effects were found after 6 months and lasted even after 1 year, showing that chronic administration of oral $1 \mathrm{~g}$ citicoline is remarkable for obtaining the outcome. This is in line with several different previous studies that have demonstrated the need for chronic administration of this cholinergic precursor [14-16, 33, 34]. The CITIRIVAD and the CITICHOLINAGE Studies showed the efficacy of combination treatment with citicoline $1 \mathrm{~g}$ and AchEls (rivastigmine and rivastigmine or donepezil, respectively) in slowing down cognitive impairment after 3 and 9 months $[14,15]$. Our remarkable starting points were the VITA and IDEALE studies [46, 47], where we demonstrated the effects of parenteral ( $2 \mathrm{~g}$ intravenously) and oral ( $1 \mathrm{~g})$ administration of citicoline, respectively in vascular cognitive impairment.

Overall, the CITIDEMAGE study has a characteristic that makes it different from all previous and recent studies on combined treatment with citicoline in dementia patients: this is the first study showing the improvement of mood and quality of life perceived with combination therapy in people with AD. Alvarez-Sabin et al. first showed that long-term treatment ( 2 years) with $1 \mathrm{~g}$ oral citicoline significantly improved the cognitive status $(p=0.005)$ and even the quality of life assessed through the EuroQoL-5D after a first ischemic stroke $[48,49]$.

The putative reasons for explaining why citicoline acts on cognition, mood, and quality of life in $A D$ patients may be substantially assumed to be the following three factors:

1. It is neuroprotective due to the activation of sirtuin, as well as other important factors such as mitochondria membrane stabilization, anti-apoptotic role, and synthesis of membrane phospholipids. Sirtuin-1 is a NAD-dependent protein deacetylase that regulates adult hippocampal neural stem cells, reduces $A \beta$ plaques and $A \beta$ amyloid, activates the transcription of the good secretase, ADAM-10 (a protein with $\alpha$-secretase activity for proteolytic processing of amyloids), and inhibits NF-k $\beta$, a protein complex that controls DNA transcription and is implicated in synaptic plasticity and memory [36, 38, 39];

2. Its metabolic pathway leads to S-adenosyl-methionine, which is a precursor of serotonin and increases the intra-synaptic levels of acetylcholine, noradrenaline, and dopamine [34]; 
3. The improved mood might be considered a key factor for the perceived quality of life, henceforth this was the first-ever study showing improved EuroQoL scores after combination treatment with citicoline plus memantine plus an AchEI.

Citicoline combined with an AchEl and memantine together could not only potentiate cholinergic neurotransmission but work as possible excitatory amino acid transporter activator (EAAT2), thus reducing the glutamate efflux [50]. Its effects on mood have already been postulated through the above-mentioned mechanism on the increase in S-adenosyl-methionine and have been confirmed by a recent study that focused on post-stroke patients [51]. This article showed the effects of cholinergic precursors, such as citicoline, on post-stroke depression through its pleiotropic mechanisms [51].

However, the CITIDEMAGE study presents strong limitations:

1) sample size limited to a few patients;

2) kind of the study, retrospective, unblinded, and observational;

3) the need for further confirmation for our hypotheses, which could derive from an openlabel, blinded, and wider study. We strongly argue that this old drug has been misused so far, especially because of unproportionate expectations from short-time administration.

4) the presence of a possible recall bias due to the observational and retrospective nature of the study.

\section{Conclusions}

In light of the importance of maximizing the pharmacological means in $A D$, the present study encourages the role of combined administration of AchEls plus memantine plus citicoline in disease management. This is one of the first studies assessing the effectiveness of combination treatment with citicoline after 12 months. Furthermore, unlike certain previous studies or even recent studies that have tried to focus on the effectiveness of citicoline, this is one of the first studies assessing the benefits on the mood and quality of life perceived. Our study showed an improvement in mood and the quality of life, both at 6 months and 1 year.

The identification of biomarkers for $A D$ enables early detection of the disease and could accelerate the development of new treatments. In the future, more will be understood about the most effective therapies against AD. In the meanwhile, our duty as medical doctors taking care of patients affected with dementia is to try to optimize the available treatments. To this purpose, using all the drugs available, including combination treatment with citicoline, appears a reasonable tool to slow down the disease progression.

\section{Author Contributions}

Study concept and design: Dr. Pietro Gareri, Dr. Antonino Maria Cotroneo, Dr. Salvatore Putignano. Acquisition of data: Dr. Pietro Gareri, Dr. Antonino Maria Cotroneo, Dr. Giuseppe Orsitto, Dr. Salvatore Putignano. Analysis and interpretation of data: Dr. Pietro Gareri and Dr. Nicola Veronese. Drafting of the manuscript: Dr. Pietro Gareri and Dr. Nicola Veronese. Critical revision of the manuscript: Dr. Pietro Gareri and Dr. Nicola Veronese. 


\section{Competing Interests}

None. Dr. Pietro Gareri, Dr. Antonino Maria Cotroneo, Dr. Giuseppe Orsitto, Dr. Nicola Veronese and Dr. Salvatore Putignano have no conflict of interest to declare for the present study.

\section{References}

1. Evans DA, Funkenstein HH, Albert MS, Scherr PA, Cook NR, Chown MJ, et al. Prevalence of Alzheimer's disease in a community population of older persons: Higher than previously reported. JAMA. 1989; 262: 2551-2556.

2. Hebert LE, Weuve J, Scherr PA, Evans DA. Alzheimer disease in the United States (2010-2050) estimated using the 2010 census. Neurology. 2013; 80: 1778-1783.

3. Alzheimer's Association. 2020 Alzheimer's disease facts and figures. Alzheimers Dement. 2020; 16: 391-460.

4. Birks JS. Cholinesterase inhibitors for Alzheimer's disease. Cochrane Database Syst Rev. 2006. Doi: 10.1002/14651858.CD005593.

5. Areosa SA, Sherriff F, McShane R. Memantine for dementia. Cochrane Database Syst Rev. 2006. Doi: 10.1002/14651858.cd003154.pub5.

6. Winblad B, Jones RW, Wirth Y, Stöffler A, Möbius HJ. Memantine in moderate to severe Alzheimer's disease: A meta-analysis of randomised clinical trials. Dement Geriatr Cogn Disord. 2007; 24: 20-27.

7. Parsons CG, Danysz W, Dekundy A, Pulte I. Memantine and cholinesterase inhibitors: Complementary mechanisms in the treatment of Alzheimer's disease. Neurotox Res. 2013; 24: 358-369.

8. Nyakas C, Granic I, Halmy LG, Banerjee P, Luiten PG. The basal forebrain cholinergic system in aging and dementia. Rescuing cholinergic neurons from neurotoxic amyloid- $\beta 42$ with memantine. Behav Brain Res. 2011; 221: 594-603.

9. Gareri P, Putignano D, Castagna A, Cotroneo AM, De Palo G, Fabbo A, et al. Retrospective study on the benefits of combined memantine and cholinesterase inhibitor treatment in aged patients affected with Alzheimer's disease: The MEMAGE study. J Alzheimers Dis. 2014; 41: 633640.

10. Carriba $\mathrm{P}$, Comella JX. Neurodegeneration and neuroinflammation: Two processes, one target. Neural Regen Res. 2015; 10: 1581-1583.

11. Hampel H, Mesulam MM, Cuello AC, Khachaturian AS, Vergallo A, Farlow MR, et al. Revisiting the cholinergic hypothesis in Alzheimer's disease: Emerging evidence from translational and clinical research. J Prev Alzheimers Dis. 2019; 6: 2-15.

12. Alexander GC, Emerson S, Kesselheim AS. Evaluation of aducanumab for Alzheimer disease: Scientific evidence and regulatory review involving efficacy, safety, and futility. JAMA. 2021; 325: 1717-1718.

13. Amenta F, Carotenuto A, Fasanaro AM, Rea R, Traini E. The Ascomalva trial: Association between the cholinesterase inhibitor donepezil and the cholinergic precursor choline alphoscerate in Alzheimer's disease with cerebrovascular injury: Interim results. J Neurol Sci. 2012; 322: 96-101.

14. Castagna A, Cotroneo AM, Ruotolo G, Gareri P. The Citirivad study: Citicoline plus rivastigmine in elderly patients affected with dementia study. Clin Drug Investig. 2016; 36: 1059-1065. 
15. Gareri P, Castagna A, Cotroneo AM, Putignano D, Conforti R, Santamaria F, et al. The citicholinage study: Citicoline plus cholinesterase inhibitors in aged patients affected with Alzheimer's disease study. J Alzheimers Dis. 2017; 56: 557-565.

16. Gareri P, Cotroneo AM, Orsitto G, Putignano S. The Citimem study: A pilot study. Optimizing pharmacological treatment in dementia. Arch Gerontol Geriatr. 2020; 89: 104073.

17. Castagna A, Manzo C, Fabbo A, Lacava R, Ruberto C, Ruotolo G. The Citimeriva Study: Citicoline plus memantina plus rivastigmine in older patients affected with Alzheimer's disease. Clin Drug Investig. 2021; 41: 177-182.

18. Castagna A, Fabbo A, Manzo C, Lacava R, Ruberto C, Ruotolo G. A retrospective study on the benefits of combined citicoline, memantine, and acetylcholinesterase inhibitor treatments in older patients affected with Alzheimer's disease. J Alzheimers Dis. 2021; 79: 1509-1515.

19. Dubois B, Feldman HH, Jacova C, Hampel H, Molinuevo JL, Blennow K, et al. Advancing research diagnostic criteria for Alzheimer's disease: The IWG-2 criteria. Lancet Neurol. 2014; 13: 614629.

20. Magni E, Binetti G, Bianchetti A, Rozzini R, Trabucchi M. Mini-mental state examination: A normative study in Italian elderly population. Eur J Neurol. 1996; 3: 198-202.

21. Katz S. Assessing self-maintenance: Activities of daily living, mobility, and instrumental activities of daily living. J Am Geriatr Soc. 1983; 31: 721-727.

22. Lawton MP, Brody EM. Assessment of older people: Self-maintaining and instrumental activities of daily living. Gerontologist. 1969; 9: 179-186.

23. Cummings JL, Mega M, Gray K, Rosenberg-Thompson S, Carusi DA, Gornbein J. The neuropsychiatric inventory: Comprehensive assessment of psychopathology in dementia. Neurology. 1994; 44: 2308-2314.

24. Miller MD, Paradis CF, Houck PR, Mazumdar S, Stack JA, Rifai AH, et al. Rating chronic medical illness burden in geropsychiatric practice and research: Application of the cumulative illness rating scale. Psychiatry Res. 1992; 41: 237-248.

25. Rinaldi P, Mecocci P, Benedetti C, Ercolani S, Bregnocchi M, Menculini G, et al. Validation of the five-item geriatric depression scale in elderly subjects in three different settings. J Am Geriatr Soc. 2003; 51: 694-698.

26. Balestroni G, Bertolotti GL. EuroQol-5D (EQ-5D): Uno strumento per la misura della qualità della vita. Monaldi Arch Chest Dis. 2012; 78: 155-159.

27. Espie CA, Kyle SD, Hames P, Gardani M, Fleming L, Cape J. The sleep condition indicator: A clinical screening tool to evaluate insomnia disorder. BMJ Open. 2014; 4: e004183.

28. Gareri P, Cotroneo AM, Orsitto G, Putignano S. The Citidemage study: Stressing the cholinergic hypothesis for the best outcomes in dementia patients: Human/human trials: Cognitive enhancement. Alzheimers Dement. 2020; 16: e038178.

29. Boyle LM. A neuroplasticity hypothesis of chronic stress in the basolateral amygdala. Yale J Biol Med. 2013; 86: 117-125.

30. Cacabelos $R$, Takeda $M$, Winblad $B$. The glutamatergic system and neurodegeneration in dementia: Preventive strategies in Alzheimer's disease. Int J Geriatr Psychiatry. 1999; 14: 3-47.

31. Cook S. The unique benefits of citicoline: An emerging nootropic and brain health nutrient. Natural medicine journal. King of Prussia: IMPACT Health Media, Inc.; 2018.

32. Secades JJ. Citicoline in the treatment of cognitive impairment. J Neurol Exp Neurosci. 2019; 5: 14-26. 
33. Secades JJ, Frontera G. CDP-choline: Pharmacological and clinical review. Methods Find Exp Clin Pharmacol. 1995; 17: 1-54.

34. Gareri P, Castagna A, Cotroneo AM, Putignano S, De Sarro G, Bruni AC. The role of citicoline in cognitive impairment: Pharmacological characteristics, possible advantages, and doubts for an old drug with new perspectives. Clin Interv Aging. 2015; 10: 1421-1429.

35. Secades JJ. Citicoline: Pharmacological and clinical review, 2010 update. Rev Neurol. 2011; 1; 52: S1-S62.

36. Hurtado O, Hernández-Jiménez M, Zarruk JG, Cuartero MI, Ballesteros I, Camarero G, et al. Citicoline (CDP-choline) increases $S$ irtuin1 expression concomitant to neuroprotection in experimental stroke. J Neurochem. 2013; 126: 819-826.

37. Hurtado $O$, Moro MA, Cárdenas $A$, Sánchez $V$, Fernández-Tomé $P$, Leza $J C$, et al. Neuroprotection afforded by prior citicoline administration in experimental brain ischemia: Effects on glutamate transport. Neurobiol Dis. 2005; 18: 336-345.

38. Martynov MY, Gusev El. Current knowledge on the neuroprotective and neuroregenerative properties of citicoline in acute ischemic stroke. J Exp Pharmacol. 2015; 7: 17-28.

39. Zhang F, Wang S, Gan L, Vosler PS, Gao Y, Zigmond MJ, et al. Protective effects and mechanisms of sirtuins in the nervous system. Prog Neurobiol. 2011; 95: 373-395.

40. Nalivaeva NN, Zhuravin IA, Turner AJ. Neprilysin expression and functions in development, ageing and disease. Mech Ageing Dev. 2020; 192: 111363.

41. Krupinski J, Abudawood M, Matou-Nasri S, Al-Baradie R, Petcu EB, Justicia C, et al. Citicoline induces angiogenesis improving survival of vascular/human brain microvessel endothelial cells through pathways involving ERK1/2 and insulin receptor substrate-1. Vasc Cell. 2012; 4: 20.

42. Gutiérrez-Fernández M, Rodríguez-Frutos B, Fuentes B, Vallejo-Cremades MT, Álvarez-Grech J, Expósito-Alcaide $\mathrm{M}$, et al. CDP-choline treatment induces brain plasticity markers expression in experimental animal stroke. Neurochem Int. 2012; 60: 310-317.

43. Secades JJ, Lorenzo JL. Citicoline-pharmacological and clinical review, 2006 update. Methods Find Exp Clin Pharmacol. 2006; 28: 1-56.

44. García-Cobos R, Frank-García A, Gutiérrez-Fernández M, Díez-Tejedor E. Citicoline, use in cognitive decline: Vascular and degenerative. J Neurol Sci. 2010; 299: 188-192.

45. Jasielski P, Piędel F, Piwek M, Rocka A, Petit V, Rejdak K. Application of citicoline in neurological disorders: A systematic review. Nutrients. 2020; 12: 3113.

46. Cotroneo AM, Castagna A, Putignano S, Lacava R, Fantò F, Monteleone $F$, et al. Effectiveness and safety of citicoline in mild vascular cognitive impairment: The ideale study. Clin Interv Aging. 2013; 8: 131-137.

47. Putignano S, Gareri P, Castagna A, Cerqua G, Cervera P, Cotroneo AM, et al. Retrospective and observational study to assess the efficacy of citicoline in elderly patients suffering from stupor related to complex geriatric syndrome. Clin Interv Aging. 2012; 7: 113-118.

48. Alvarez-Sabín J, Ortega G, Jacas C, Santamarina E, Maisterra O, Ribo M, et al. Long-term treatment with citicoline may improve poststroke vascular cognitive impairment. Cerebrovasc Dis. 2013; 35: 146-154.

49. Alvarez-Sabín J, Santamarina E, Maisterra O, Jacas C, Molina C, Quintana M. Long-term treatment with citicoline prevents cognitive decline and predicts a better quality of life after a first ischemic stroke. Int J Mol Sci. 2016; 17: 390. 
50. Lin $\mathrm{CL}$, Kong $\mathrm{Q}$, Cuny GD, Glicksman MA. Glutamate transporter EAAT2: A new target for the treatment of neurodegenerative diseases. Future Med Chem. 2012; 4: 1689-1700.

51. Arcadi FA, Corallo F, Torrisi M, Scarfi C, Lo Buono V, Formica C, et al. Role of citicoline and choline in the treatment of post-stroke depression: An exploratory study. J Int Med Res. 2021; 49: 03000605211055036.



Enjoy OBM Geriatrics by:

1. Submitting a manuscript

2. Joining in volunteer reviewer bank

3. Joining Editorial Board

4. Guest editing a special issue

For more details, please visit:

http://www.lidsen.com/journals/geriatrics 